\section{Magnetic Rotatory Dispersion and Absorption of the Cerous Ion in Solution}

Is a previous communication ${ }^{1}$, we pointed out that in order to explain our results on the magnetic rotation of solutions of cerous sulphate in water, it was necessary to use two absorption wave-lengths in the Ladenburg paramagnetic rotation formula, namely, $2960 \mathrm{~A}$. (actual) and $2390 \mathrm{~A}$. (effective). This latter value seemed reasonable, as intense absorption bands were located with wave-lengths 2540,2400 and $2230 \mathrm{~A}$. in aqueous solutions of cerous sulphate.

We have extended our work to solutions of greater concentration, and have confirmed our previous experimental results. However, by using Rosenfeld's quantum mechanical theory of the Faraday effect ${ }^{2}$, instead of Ladenburg's formula, we find that the rotation of the $\mathrm{Ce}^{+}++$ion throughout the range $5780-3341 \mathrm{~A}$. is, to our degree of accuracy, controlled by the two absorption bands with wave-lengths 2960 and $2540 \mathrm{~A}$. For these absorption bands the transition assignments given by Bose and Datta ${ }^{3}$ were assumed, namely, $4^{2} F_{5 / 2} \rightarrow 5^{2} D_{5 / 2}$ for $2960 \mathrm{~A}$., and $4^{2} F_{5 / 2} \rightarrow 5^{2} D_{3 / 2}$ for $2540 \mathrm{~A}$. For $\lambda 3128 \mathrm{~A}$., however, we find that the calculated value of the rotation does not agree within the limits of experimental error with the observed rotation, indicating the presence of a small effect due to the absorption bands with wave-lengths 2400 and $2230 \mathrm{~A}$.

Throughout the range $\lambda 5780-3341 \mathrm{~A}$. our calculations of the strengths ( $f$ values) for the transitions $4^{2} F_{5 / 2} \rightarrow 5^{2} D_{5 / 2}$ and $4^{2} F_{5 / 2} \rightarrow 5^{2} D_{3 / 2}$ give $1.40 \times 10^{-3}$ and $2.29 \times 10^{-2}$ respectively. These results are in fair agreement with estimates which we have made directly from absorption data for dilute solutions of cerous sulphate.

So far as order of magnitude only is concerned, our larger $f$ value is in agreement with calculations made by Gorter ${ }^{4}$ and Serber (quoted by Gorter) on different grounds.

\section{R. W. ROBERTS. \\ L. A. WALLACE. \\ I. T. PIERCE.}

George Holt Physics Laboratory, University of Liverpool. Oct. 23.

NATURE, 130, 890, Dec. 10, 1932.

Z. I'hys., 57, $835 ; 1930$.

Natire, 128, 2\%0, Aug. 15, 1931.

- Phys. Z., 34, 238; 1933.

\section{Spectral Absorption of Methylated Xanthines and} Constitution of the Purine Nucleosides

AN examination of the ultra-violet spectral absorption of xanthine and certain methylated xanthines suggests that we possess a method of distinguishing between derivatives of xanthine (isoxanthine) which are substituted in the 7 or 9 positions, and that this method may be used to assign to one or other position the carbohydrate radical in the natural and synthetic purine glycosides.

Xanthine and its methyl derivatives may be divided into two groups, as follows:

(1) Those of which the absorption curves exhibit two bands in alkaline solution, namely, xanthine, 1-, 8-, 9-monomethylxanthines, $3: 9$-dimethylxanthine, $1: 3: 9$-trimethylxanthine.

(2) Those of which the curves exhibit one band in both acid and alkaline solution, namely, 3-, 7-monomethylxanthines, $1: 3-, \quad 1: 7-, \quad 3: 7$-dimethylxanthines, and $1: 3: 7$-trimethylxanthine.

It is evident that the presence of methyl in position 7 inhibits the appearance of the second band. A comparison of the spectra of methylated purines with those in which the hydrogen of the imino-group of the glyoxaline ring is unsubstituted suggests that in 3-methylxanthine and in theophylline (methyl groups in positions 1 and 3 ) this hydrogen is attached at position 7, whereas xanthine and 1- and 8-methylxanthines have the hydrogen linked to position 9, the reverse of the arrangement usually assigned to these compounds. The formulæ of xanthine and the hypothetical isoxanthine would therefore have to be interchanged.<smiles>O=C1NC(=O)C2N=CNC2N1</smiles>

Xanthine

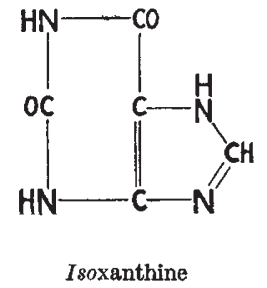

Isoxanthine
The spectrum of xanthosine (xanthine riboside), prepared from guanosine from yeast nucleic acid, shows two bands in alkaline solution, the maxima occurring at the same wave-lengths as those of 9methylxanthine. In contrast to this, theophylline$d$-glueoside ${ }^{1}$ and theophylline- $l$-arabinoside ${ }^{2}$, both prepared synthetically, show only one band, and their absorption curves are identical with that of caffeine.

It thus appears probable that in xanthosine from yeast nucleic acid the carbohydrate radical is attached in position 9 , whereas the synthetic arabinoside and glucoside of theophylline contain the sugar in position 7. The synthetic glycosides are prepared by the action of the acetylated bromo-derivative of the sugar on the silver salt of the purine, and the location of the carbohydrate group in position 7 is in harmony with the conversion of silver theophylline into caffeine by means of methyl halides.

The conclusions now drawn as to the positions of the carbohydrate radicals in the natural nucleosides are opposed to those of Gulland and Macrae ${ }^{3}$. These were based on what appears to be less satisfactory evidence than that now presented, and it is hoped that an examination of the spectra of methylated xanthosines will allow a decision to be reached. This work is in progress.

A full account of these investigations will be published later, and we intend to extend the method to the examination of the position of the carbohydrate radicals in other nucleosides.

Biochemical Department,

J. M. GuLuand.

The Lister Institute, London, S.W.1.

The Medical Unit,

The London Hospital, London, E.1. Oct. 5 .

E. R. Holiday.

3 Fischer and Helferich, Ber., 47, 210 ; 1914.

2 Pryde and Williams, J. Chem. Soc., 640; 1933.

J. Chem. Soc., 662; 1933. 